\title{
All-optical silicon simplified passive modulation
}

\author{
R. Aharoni \\ Faculty of Engineering, Bar-Ilan University, Ramat-Gan 52900, Israel \\ Bar-Ilan Institute for Nanotechnology \& Advanced Materials, Ramat-Gan 52900, Israel \\ Physics department, Bar-Ilan University, Ramat-Gan 52900, Israel \\ O. Baharav \\ Faculty of Engineering, Bar-Ilan University, Ramat-Gan 52900, Israel \\ L. Bidani \\ Faculty of Engineering, Bar-Ilan University, Ramat-Gan 52900, Israel \\ M. Sinvani \\ Faculty of Engineering, Bar-Ilan University, Ramat-Gan 52900, Israel \\ Bar-Ilan Institute for Nanotechnology \& Advanced Materials, Ramat-Gan 52900, Israel \\ D. Elbaz \\ Faculty of Engineering, Bar-Ilan University, Ramat-Gan 52900, Israel

\section{Z. Zalevsky} \\ Faculty of Engineering, Bar-Ilan University, Ramat-Gan 52900, Israel \\ Bar-Ilan Institute for Nanotechnology \& Advanced Materials, Ramat-Gan 52900, Israel
}

In this paper we present an all-optical silicon based modulator suggested also for high power operation and for pulse picker application being used as part of fiber lasers system. The paper theoretically and experimentally investigates several new and important insights involving the dependence of the relative transmission on the pump pulse energy for different finesse values of the constructed cavity as well as the dependence of the response rate of the device to the pump wavelength due to coexistence of two physical recombination processes: fast surface effect and slow bulk recombination. To adapt the constructed silicon based cavity to be used in lasers applications, we aligned the pump and the signal beams to co-propagate through the device while the usage of a cavity allowed a low power pump to yield a significant extinction ratio at the output of the device.

[DOI: http://dx.doi.org/10.2971/jeos.2012.12029]

Keywords: optical modulator, silicon, cavity

\section{INTRODUCTION}

The realization of fiber integrated all-optical modulators has high importance in the field of optics communication that is seeking for fast, cheap and integrated ways to perform modulation of information carried over the 1.3 and $1.55 \mu \mathrm{m}$ wavelength $[1,2]$.

All-optical modulation is realized when a transmission of a certain light beam is controlled by the presence of another light beam. This type of modulation may achieve higher modulation rates by avoiding hardware performing conversion into electronic domain and then back to photons. Avoiding such hardware makes the modulation process faster, less noisy and less cumbersome. Since a direct modification of the absorption coefficient cannot perform a direct significant modulation and the desire is to use silicon for integrated photonics applications (see Section 2), there were numerous attempts to use different configurations of interferometric modulation devices or by using special electronic junctions. In 2004 Intel research group introduced for the first time silicon integrated modulator operating at modulation rates $>1 \mathrm{GHz}$ based on a metal oxide semiconductor (MOS) capacitor as a Mach Zehnder interferometer (MZI) [3]. One year later they introduced a faster device with similar basic operation mech- anism that exceeded $10 \mathrm{GHz}$ and included 11 sections of electrodes that allowed applying external DC bias voltage as well as AC voltage command over $4 \mathrm{~mm}$ interaction length [4]. The modulation rate was further enhanced by Passaro [5] who suggested MOS-capacitor-based SOI optical modulator with $14.36 \mathrm{GHz}$ bandwidth based on an optoelctronic model investigating different architectures for silicon plasma dispersion modulators as well as for fitting to experimental results reported in the literature. Lipson et al predicted [6] and demonstrated [7] optical modulation through light-confining resonating structures to enhance the effect of refractive index change on the transmission response. In this device decreasing its size reduces the power requirement without increasing the $\mathrm{Q}$ factor of the device and managed to reduce its modal volume to $6 \mu \mathrm{m}$. Ultrafast silicon on insulator (SOI) all optical modulators which require only $6 \mathrm{fJ}$ and operate with response rate of only few ps [8] have also been recently suggested. Reed provided a short review of some prominent silicon based modulators [9]. These devices are realized through the electro-optic world of semiconductors, governed by planar complex structures often on top of 3D structures containing metals and insulator materials and its features are in the nanometer regime arranged tightly on a chip - which makes 
them very expensive and hard to produce. It is also not adequate to have them integrated in fiber systems such as fiber lasers, communication systems etc.

Another advantage of our proposed device is the fact that it is completely passive and does not consume electrical power to control the carriers lifetime such as p-i-n junction e.g. in Ref. [10]. This for example, makes is suitable for an all-fiber configuration.

An interesting and relevant approach was previously proposed by Narayanan [11] who suggested using hydrogenated amorphous-silicon in order to accelerate the operation speed, since then the free-carrier mobility is lower. In that configuration, similarly to Ref. [2] and in contrast to this paper, the pump is incident on top of the waveguide, and not copropagating with the modulated signal. High power fiber lasers are one of the most rapidly growing fields of research due to their applicability not only to the field of optics communication but also their importance in fields as home land security, medicine and processing of materials. The recent advance in this field allowed realization of very high power fiber lasers at very large spectral range starting from the UV and up to the infra-red, some can produce several KW at their output [12]-[18].

This paper presents the construction of the main building block for a fiber integrated modulation device suitable also for relatively high power fiber laser applications in which fast silicon based all-optical (AO) device is integrated into a fiber based configuration to realize a modulator, pulse picker or Q-switch. In such a device, the signal (i.e. the modulated laser) and the pump are co-propagating through the suggested pulse picker and the pump laser is absorbed to control and attenuate the signal. This can be realized through an in fiber device where a short section of the fiber is made of silicon cavity, or a free space device in a standard free space cavity laser.

The capabilities of the proposed device can also be used for Q-switching and pulse picking applications, or any application that utilizes fast switching between low and high optical losses. These applications, realized via an AO modulator, has an important role in the field of high power fiber laser while being the module responsible for reducing and controlling the laser's pulse repetition rate. A built in AO pulse picker allows internal, step-wise changes in the repetition rate. An AO pulse picker in the external laser head may enable selection of variable, shaped pulse trains at varied repetition rates from a single-shot and up to a burst-mode operation.

Note that pulse picking, for example, is required in $\mathrm{Yb}$-doped fiber lasers in order to reduce the rate of the output pulses from rates of $\mathrm{MHz}$ into the range of $\mathrm{KHz}$. Current state-of-theart technologies involve the use of acousto-optic modulators (AOM) or electro-optics modulators (EOM). For the specific system into which our modulator can be integrated the technical requirements for the pulse picker involve response rate (rise and fall time) of below 10nsec, modulation frequency of less than $1 \mathrm{MHz}$, extinction ratio of $20 \mathrm{~dB}$ and insertion losses of below $1 \mathrm{~dB}$, and the proposed device can fulfill these require- ments as seen in the experimental section of this paper - the fall time in this paper is limited by the pump pulse and can get shorter to pico-second scale, and also the recovery time can be further reduced beyond the presented rates, as suggested in Section 2.2.

In Sections 2.2 and 2.3 we further explain the reasons for using silicon rather than realizing an $\mathrm{AO}$ modulator based on more "optically friendly" materials. The main reasons are the fabrication costs, capabilities, low non linearities and techniques coming from the microelectronics industry are well adapted to silicon, and the global desire to incorporate microelectronic circuitry that will be integrated with the AO modulator and which will allow performing further processing of signals and information. Another important feature of an in-fiber device is the environmental stability (discussed in the theoretical section) and should not include free-space optics sections which also make the system more cheap and compact. Nevertheless it is important to stress out that our main motivation to construct an all-optical silicon based modulator is the related capability to integrate in the silicon layer that is used for the photonic modulation, also micro-electronic circuitry performing control of the overall laser system (thermal controller etc). A realization of micro controller integrated with the photonic device is a new and an applicable direction that has not been previously explored. Furthermore, the proposed device is compatible with common fiber lasers techniques like Yb-doped fiber lasers, mostly operate around 1050 - $1100 \mathrm{~nm}$. As shown in this paper, a thin silicon layer can generate high modulation depth, and for $1070 \mathrm{~nm}$ signal, which is on the silicon absorption edge, the absorption loss is $\sim 0.1 \mathrm{~dB}$ for a 30 $\mu \mathrm{m}$ silicon layer. This makes the proposed technique appealing to the field of fiber lasers.

The novelties of the device introduced in this paper include the combination of several aspects advantages. First, we provide an experimental thoroughly investigation of the physical processes of recombination occurring in the silicon layers and specifically we demonstrate the dependence of the device response rate (i.e. the modulation frequency) on the pump wavelength. A technique for separating the bulk and surface recombination has been suggested for example by Gaubas \& Vanhellemont [19], due to its crucial relevance on the device operation rates. However, we were able to experimentally implement and demonstrat the models regarding the difference between the surface versus the bulk recombination process in silicon. Second, in order to adapt the AO silicon based configuration to high power fiber lasers application, or more accurately speaking to laser powers being higher than the typical power level used in optics communication applications, we constructed a cavity in which the pump and the signal are copropagated through the device. Due to the fact that we used a cavity a low power pump could generate significant extinction ratio. In this paper we experimentally validated the dependence of the relative transmission of the constructed device on the pump pulse energy for different finesse values of the constructed cavity. We also showed its connection to the theoretical general plasma dispersion relation. Thus, this devise is adaptable of the proposed fiber integrated compatible configuration to optical fibers configurations operating at optical power level higher than the one used in optics communi- 
cation applications. Another advantage is that the proposed device built from silicon and thus is suitable for operation at the output of $\mathrm{Yb}$-doped fiber lasers while modulating signal at wavelength of about $1070 \mathrm{~nm}$ (a wavelength for which most of the optics communication modulation devices are not suitable, and the usage of silicon as the optical modulation substrate within hybrid and integrated modulation device. This eventually yields compatibility to micro-electronics fabrication facilities as well as an integration of required microelectronic processing circuitry (e.g. micro controller to control the performance of the proposed modulator) on the same silicon layer being used for the photonic modulation.

\section{THEORY}

\subsection{All-optical modulation}

The plasma dispersion effect has been deeply studied [1] and used for various optical modulation and light controlling devices $[2,20,21]$. According to the current theory, based on the Drude model [22], photons with wavelengths having above band gap energy and which are being illuminated upon a semi-conductor, will be absorbed to create electron-hole pairs (EHP) that will modify the optical properties of the semiconductor according to the following relations:

$$
\begin{aligned}
& \Delta \alpha=\frac{e^{3} \lambda^{2}}{4 \pi^{2} c^{3} \varepsilon_{0} n}\left[\frac{\Delta N_{e}}{\mu_{e} m_{e}^{* 2}}+\frac{\Delta N_{h}}{\mu_{h} m_{h}^{* 2}}\right] \\
& \Delta n=\frac{-e^{2} \lambda^{2}}{8 \pi^{2} c^{2} \varepsilon_{0} n}\left[\frac{\Delta N_{e}}{m_{e}^{*}}+\frac{\Delta N_{h}}{m_{h}^{*}}\right]
\end{aligned}
$$

where $\Delta \alpha$ is the change in the absorption coefficient (the absorption coefficient is related to the imaginary part of the index of refraction by $n=n_{R}+i \frac{\alpha \lambda}{4 \pi}, \lambda$ is the wavelength, $n$ is the refraction index, $\mu_{e}$ and $\mu_{h}$ are the mobility of electrons and holes respectively, $m_{e}^{*}$ and $m_{h}^{*}$ are the effective masses of electrons and holes respectively, $\varepsilon_{0}$ is the dielectric constant, $c$ is the speed of light in vacuum, $e$ is the charge of an electron and $\Delta \mathrm{N}_{e}$ and $\Delta \mathrm{N}_{h}$ are the change in the concentration of free electrons and holes in the semiconductor, respectively. These relations were later determined experimentally as [23]:

$$
\begin{aligned}
& \Delta \alpha=-8.5 \cdot 10^{-18} \Delta N_{e}-6 \cdot 10^{-18} \Delta N_{h} \\
& \Delta n=-8.8 \cdot 10^{-22} \Delta N_{e}+6 \cdot 10^{-18} \Delta N_{h}
\end{aligned}
$$

However, the current theory is valid for charge carrier densities between $10^{17}$ to $10^{20} \mathrm{~cm}^{-3}$, and above this concentration this theory is not valid. In addition, this theory was not validated for all possible combinations of wavelengths for the pump and the signal, only for $1.5 \mu \mathrm{m}$ and $1.3 \mu \mathrm{m}$. One can see that the modulation abilities of this effect are limited, and high values of absorption are not feasible since generating densities higher than $10^{20} \mathrm{~cm}^{-3}$ is not realistic.

An efficient and common method of enhancing weak optical effects is using an optical cavity. In the suggested device the cavity employs not only the change in the absorption coefficient $(\Delta \alpha)$ but also shows a strong sensitivity of the cavity to small phase changes resulting from a fractional changes in the refraction index $(\Delta \mathrm{n})$, as the transmission function is spectrally shifted and attenuated by the increased absorption due to the change in the refraction index.
Fabry-Perot transmission ( $R=30 \%)$

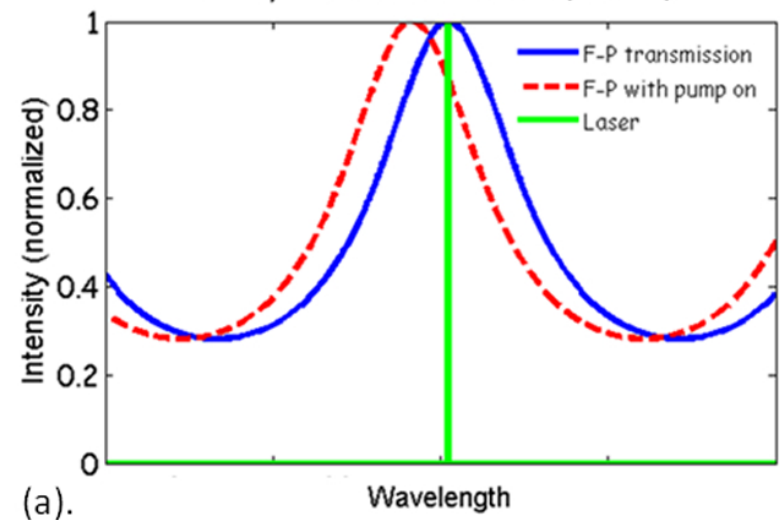

Fabry-Perot transmission ( $R=95 \%)$

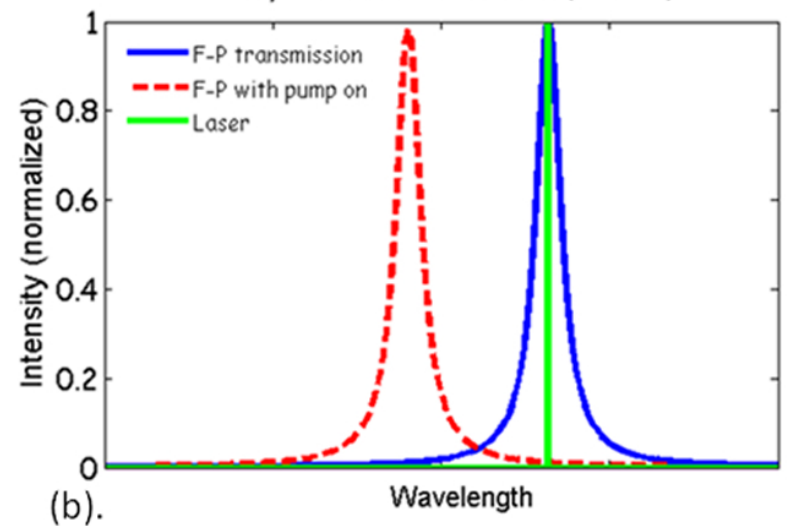

FIG. 1 Numerical simulations. (a). Transmission of F-P cavity $(R=0.3)$ with and without the pump beam. The spectral bandwidth of the laser is very narrow and it seems like a delta function. (b). Transmission of F-P cavity $(R=0.95)$ with and without the pump beam.

The transmission of Fabry-Perot (F-P) interferometer consisting from a lossy (or a gain) media, with refraction index $n$, between the two mirrors (with equal reflectivity $\mathrm{R}$, and with separation distance of $\mathrm{L}$ ) and in which we denote the intensity gain coefficient per pass as $X$, becomes:

$$
\frac{I_{O u t}}{I_{I n}}=\frac{X(1-R)^{2}}{(1-R X)^{2}+4 R X \sin ^{2}\left(\frac{\delta}{2}\right)}
$$

where $\delta$ is the round-trip phase equals to $\delta=2 \mathrm{kLn}$ (where $k=2 \pi / \lambda)$. The derivation of Eq. (3) differs from the usual F$\mathrm{P}$ interferometer only in adding constant gain factor to each round-trip. We can use the Beer-Lambert law for the absorption term and generalize the previous equation for an asymmetric lossy F-P cavity as follows:

$$
\frac{I_{\text {Out }}}{I_{\text {In }}}=\frac{\left(1-R_{1}\right)^{2}\left(1-R_{2}\right)^{2} e^{-\alpha L}}{\left(1-\sqrt{R_{1}} \sqrt{R_{2}} e^{-\alpha L}\right)^{2}+4 \sqrt{R_{1}} \sqrt{R_{2}} e^{-\alpha L} \sin ^{2}\left(\frac{\delta}{2}\right)}
$$

where $R_{1}$ and $R_{2}$ are the first and second mirrors reflectivity coefficients respectively and $\alpha$ is the absorption coefficient (in $\mathrm{cm}^{-1}$ ). Eq. (4) is to be used when the pump illumination is on, therefore we must consider the $\Delta \mathrm{n}$ contribution over the penetration depth of the pump so the total phase is actually being related to $L, L_{p}$ and $\Delta \mathrm{n}$, where $L_{p}$ is the penetration depth of the pump wavelength and the effective optical length $\left(L_{o p}\right)$ becomes $L_{o p}=L n+L_{p} \Delta n$. 
For all the experiments presented in this paper we used a narrow band infra-red (IR) source (produced by tunable laser HP 8168 F) with $100 \mathrm{KHz}$ bandwidth amplified by an EDFA, and a $500 \mu \mathrm{m}$ thick double side polished intrinsic silicon $(\sim 1700 \Omega \mathrm{cm})$ as a resonator In such a configuration modulation can be achieved even when the silicon absorption is negligible. For example, by illuminating the polished parallel silicon bulk with relatively narrow (i.e. relative to the F-P peak) band IR laser (signal beam) results with a low finesse F-P resonator $(\mathrm{f} \approx 4)$. As in every F-P resonator one can theoretically get a $100 \%$ transmission, particularly in the case of low finesse where the transmission peak is wider than the laser. When a pump pulse is introduced the absorption term can be ignored as the peak transmission of such a cavity is about one percent lower than without the pump, and the main modulation effect is caused due to the modification of the refractive index. Usage of $532 \mathrm{~nm}$ laser as a pump, results with a "shift" of the transmission function of the cavity and thus the transmission for the wavelength of the signal laser will decreased. A $50 \mu \mathrm{J}$ pump pulse will shift the transmission peak and results with $86 \%$ transmission of the device as depicted in the simulation of Figure 1(a). In this case the absorption is so low such that for a single pass more than $99 \%$ of the energy is transmitted.

If we increase the reflectivity of the surfaces to $95 \%$ to get a high finesse, the modulation due to the phase change is much stronger. Although single pass loss is magnified by the finesse (which is about 20 times larger in comparison to the previous case), the maximum transmission is still very high and the refractive index modification is still much more significant as can be seen in Figure 1(b) where theoretical modulation depth of $99 \%$ is achieved.

An important note is related to the fact that the AO modulator we are constructing can be aimed towards the pulse-picking which is being used in ultrafast laser systems where the optical spectral bandwidth of the input pulses is normally large ( $>$ a few nm). That means the use of a high-Q optical cavity in the beam path is not a good idea. In this case it is obvious that one should use relatively low finesse cavity (a finesse of about 10-20) which allows the required bandwidth of few $\mathrm{nm}$. Usage of a silicon layer (thinner than the one suggested in Figure 1) separating the reflecting mirrors of the cavity is also good not only due to response rate reasons but also in order to create larger transmission bandwidth (the smaller the separation distance is, the larger is the transmission bandwidth for a given finesse). Such a cavity can be realized by growing a thin silicon layer on top of a mirror having the desired reflectivity.

\subsection{Modulation rates in silicon}

Fast optical modulators have been previously introduced, as fast as $40 \mathrm{~Gb} / \mathrm{s}$ made of lithium niobate or III-V semiconductors. However, as mentioned before, silicon photonics is of great interest these days [24]. Due to the long history of this material in the electronic industry turn it to be well characterized, cheap, and widely used. The future integration of electronic and photonic is also of great concern and has many benefits, as a result this topic has been under intense study in the recent decades and silicon based modulators, amplifiers Raman lasers etc showed significant improvement in their ob- tainable set of performance [25]. However, the urge of using silicon is challenging since the only significant optical effect in silicon is the plasma dispersion effect as it posses weak Kerr effect, as mentioned in Ref. [22] and approximated by Ref. [1], and therefore is impractical [25]. In Silicon, as in centrosymmetric crystals, Pockels as well as Franz-Keldysh effects do not exist there $[1,26]$. One of main points of interest is the lifetime of the excited carriers, which is the only way of affecting the modulation depth and controlling the rate. Generally speaking, we can regard the EHP generation process as a very fast, almost instantaneous, process in relation with the recombination rates. Without getting into details of the recombination mechanisms, it can be easily shown that these processes (i.e. generation and recombination) are not symmetrical [27]: the generation process is a hyperbolic tangent of the light intensity $-\Delta N_{e / h} \propto \tanh (t \sqrt{\gamma \beta \alpha I})$ and the recombination is described by a much slower hyperbola for quadratic recombination (i.e. the rate of recombination is proportional to the square of the non equilibrium density) $-\Delta N_{e / h} \propto \frac{1}{(t \sqrt{\gamma \beta \alpha I}+1)}$ or decreasing exponential for linear recombination (i.e. the rate of recombination is proportional to the first power of the non equilibrium density) $\Delta N_{e / h} \propto \tau \beta \alpha I e^{-t / \tau}$, where $t$ is time, $I$ is the intensity of light, $\alpha$ is optical absorption coefficient, $\beta$ is the quantum yield, $\tau$ is the effective electron lifetime, and $\gamma$ is the recombination coefficient defined as the (average) product of the velocities of holes and electrons and the recombination cross-section.

More specifically, one must refer to the difference between bulk recombination (where recombination through traps is irrelevant in intrinsic material) which is rather slow as much as a few $\mu \mathrm{s}$, and surface recombination which is much faster and can approach a few ps [28]. Thus, in order to produce a fast device a short wavelength pump source must be used in order to control and to lower the penetration depth such that the dominant effect will be related to the fast surface processes rather than the slow bulk related interaction.

In the experimental part it will be shown that the relaxation time is representing the free carriers lifetimes due to the different recombination mechanisms. Enhancing the surface recombination processes, which results in several hundreds of picoseconds of carriers lifetime [29], by creating larger amount of carriers generated on the surface can shorten the overall lifetime. Usage of poly-silicon, or ion-implanted Si samples can further reduce the lifetime to the picoseconds regime $[11,30,31]$.

Let us for a minute discus some response time consideration in the proposed silicon based AO aiming to be used as a pulse picker. Standard mode-locked lasers normally operate with repetition rate in the range $50-100 \mathrm{MHz}$. This makes the distance between pulses of about 10-20 ns. It is thus required that the rise and fall times of the pulse-picker have to be around $10 \mathrm{~ns}$ to be able to pick out an individual pulse at a time. As previously mentioned the surface effects of silicon are fast enough to cope with this working point but the bulk response time (for instance due to diffusion and not due to drift) which are of order of several micro seconds are not. It is important to note that one of the advantages of using silicon is to have 
the ability to also integrate a micro-electronic circuitry on it. Such circuitry can be used to construct a micro controller that will stabilize and control the operability of the modulator and other related module but also be used to install voltage probes that can drift away the generated free carriers (the drift response time of silicon is much smaller than the micro second range).

\subsection{Additional Silicon considerations}

For operating as an in-cavity pulse picker the device should be capable to withstand high power pulses at the operating wavelength, i.e. the signal, as well as for the pump. The high energy laser induced damage threshold at $1550 \mathrm{~nm}$ is discussed and measured experimentally by Cowan [32]. Generally speaking, the damage threshold (and also its thermal conductivity) in silicon is $\sim 10$ times higher than in GaAs, which makes it a good optical material [24]. However, silicon posses some nonlinearities that have to be considered in a high signal power. Since silicon has an indirect bandgap of $1.12 \mathrm{eV}$ and a direct one of $3.4 \mathrm{eV}$ [33] at wavelengths used in optical communication a single photon does not have sufficient energy to excite an electron from the valance band to the conduction band. The sum of the energies from two photons is also insufficient to excite an electron across the direct band-gap of silicon, but it is sufficient to excite across the indirect bandgap of silicon. Momentum conservation requires that the indirect two photon absorption (TPA) transitions be assisted by phonons, and at $1.55 \mu \mathrm{m}$ the TPA coefficient is much smaller than for InP/InGaAsP [34] which makes silicon a good choice for relatively high power applications.

The intensity transmission due to TPA (the linear absorption can be neglected at this wavelength) is inversely proportional to the length and can be reduced, since the device thickness can be reduced to a few microns, and the optical path for the resonant wavelength $(1550 \mathrm{~nm})$ is shorter:

$$
\frac{d I}{d z}=-B I^{2} \rightarrow I=\frac{1}{B z-I_{0}^{-1}}
$$

As to the pump pulse, theoretical and experimental results show that at pulse duration in the order of nanoseconds the damage threshold is $5 \mathrm{~J} / \mathrm{cm}^{2}$ at $10 \mathrm{~ns}$ pulse duration [35]. The highest power we used in our experiment was $7.5 \mathrm{~mJ}$ and the beam area was $0.07 \mathrm{~cm}^{2}$ i.e. $100 \mathrm{~mJ} / \mathrm{cm}^{2}$ which is two order of magnitude lower than the above mentioned threshold, and it can even be reduced further with higher finesse values of fabricated future cavity.

The thermo-optical effect (TOE) should be considered in the suggested device since it influences the refractive index and the phase of the light. Cocorullo and Rendina [36] also used a similar cavity structure and determined accurately the TOE in silicon at $1.5 \mu \mathrm{m}$ and used this technique to show that the thermal expansion role in the phase change is about 20 times weaker than the TOE and measured the coefficient to be: $\frac{\partial n}{\partial T}=1.86 \cdot 10^{-4} K^{-1}$ They reported temperature variation of $<7 \mathrm{~K}$ to achieve $\pi / 2$ phase shift. The temperature variation induced phase change of the cavity through the refractive index

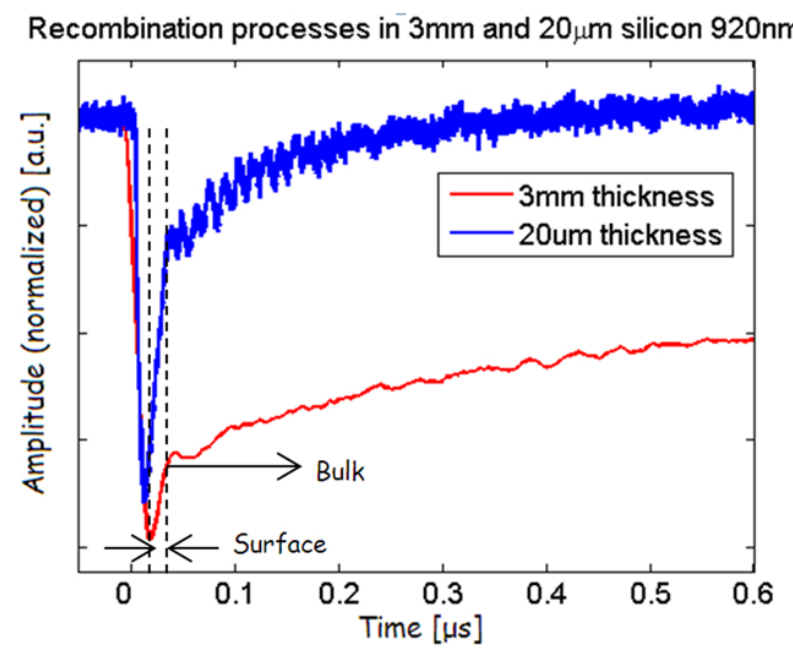

FIG. 2 Comparison of the temporal response obtained at $20 \mu \mathrm{m}$ thick (blue) and $3 \mathrm{~mm}$ thick (red) c-Si samples due to single pass absorption only. The first recombination noticed is the fast surface effect, followed by the remains of the slower bulk process.

and the thermal expansion such as:

$$
\delta \Phi=\frac{2 \pi L}{\lambda} \delta n+\frac{2 \pi n}{\lambda} \delta L=\frac{2 \pi L}{\lambda} \frac{\partial n}{\partial T} \Delta T+\frac{2 \pi n}{\lambda} \kappa L \Delta T
$$

and for our measuring system, i.e. cavity length of $\mathrm{L}=500 \mu \mathrm{m}$ (silicon linear thermal expansion coefficient $\kappa=2.6 \cdot 10^{-6} \mathrm{~K}^{-1}$, $\pi$ phase shift occurs due to temperature variation of $\sim 4 \mathrm{~K}$ (regardless the expansion coefficient) which makes the experimental system somehow unstable. This dependence can be reduced for shorter cavity lengths or by applying various thermal insulation and stabilization techniques.

Time scale should be considered too in this system but the TOE, being related to the thermal processes is rather slow and can be used for modulation at frequencies of up to $1 \mathrm{MHz}$, according to Manipatruni et al who estimated the thermal time constants to be in the order of microseconds [37].

\section{EXPERIMENTAL RESULTS}

\subsection{Preliminary results}

As a preliminary result to validate our claim regarding the above mentioned dependence of the recombination rate on the pump wavelength we used wedged n-type silicon $(\rho=0.2 \Omega \mathrm{cm})$ double side polished samples. In order to avoid interference resonance effects the samples in this experiment were wedge shaped.

The sample was illuminated with a signal laser (DFB laser at wavelength of $1550 \mathrm{~nm}$ ) while modulating it only through changing the absorption coefficient of Eq. (2) (there is no cavity in this experiment and thermo-optical effects are irrelevant) using a Surelite Continuum I-20 followed by an optical parametric oscillator (OPO). In Figure 2 one can see both processes of the bulk and surface recombination: When the pump illumination is at wavelength of $920 \mathrm{~nm}$, it penetrates dozens of microns into the bulk which results in fast recovery of the signal in thin silicon (thinner than the pump penetration depth) due to surface recombination processes and in 


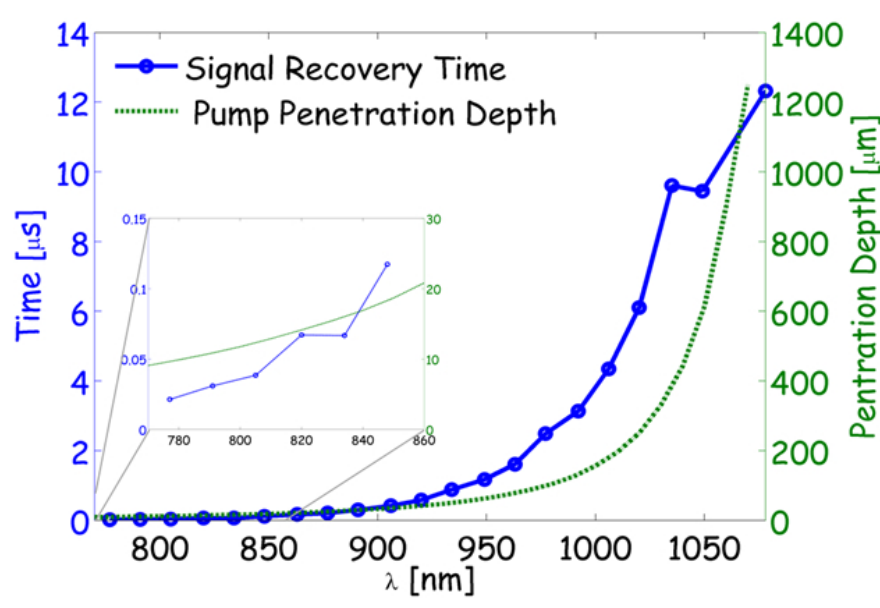

FIG. 3 Experimental results for the signal's recovery time and the penetration depth of the pump versus the wavelength of the pump. Blue - experimental results of signal's recovery time (FWHM) in comparison to the penetration depth of the pump (dashed green).

significantly slower rate of recombination due to charge carriers in the bulk. In a $3 \mathrm{~mm}$ thick sample the dominant process is the slower bulk process which results in a slow recovery, and the fast surface recombination has a small contribution. The initial fast recovery time, due to the surface recombination, can easily be distinguished from the following slower part in both samples of Figure 2 and it is limited by the pump pulse duration which was $\sim 10 \mathrm{~ns}$ in our experiment. One can see that both processes takes place in both samples, but the thinner samples exhibits more significant part of initial recovery while in the thicker one the initial fast recovery is less dominant. Note that instead of using thin silicon layer one may use thicker layer but to illuminate it with pump at shorter wavelength leading to average penetration depth of few microns.

In the experiments of Figures 3 and 4 we further demonstrate the dependence between the pump penetration depth and the recovery time of the signal, to verify the claim of temporal influence resulted from surface $\backslash$ bulk processes. As the pump wavelength decreases the less it penetrated the sample, and more charge carriers are created near the surface as the pump is decaying exponentially into the medium. Consequently, fast surface recombination processes are getting dominant as the bulk recombination effect is reduced, causing the over-all recovery time to decrease. For that we used thick silicon wedged sample (of $3 \mathrm{~mm}$ ) in order to be able to measure the change in the recovery time only due to the change in the penetration depth (there is no cavity). Figure 3 shows measured recovery time of the signal at full width half maximum (FWHM) using the $3 \mathrm{~mm}$ sample versus pump wavelength varied from $770 \mathrm{~nm}$ to $1080 \mathrm{~nm}$. One may see how the thickness of the generated free electrons-holes absorption layer affects the response time.

It also presents the experimental data of the penetration depth versus the wavelength [38]. The experimental data in Figure 3 and Figure 4 is taken from a series of measurements similar to the measurement of Figure 2, where we extracted the FWHM of the signal attenuation for every pump wavelengths. Qual-

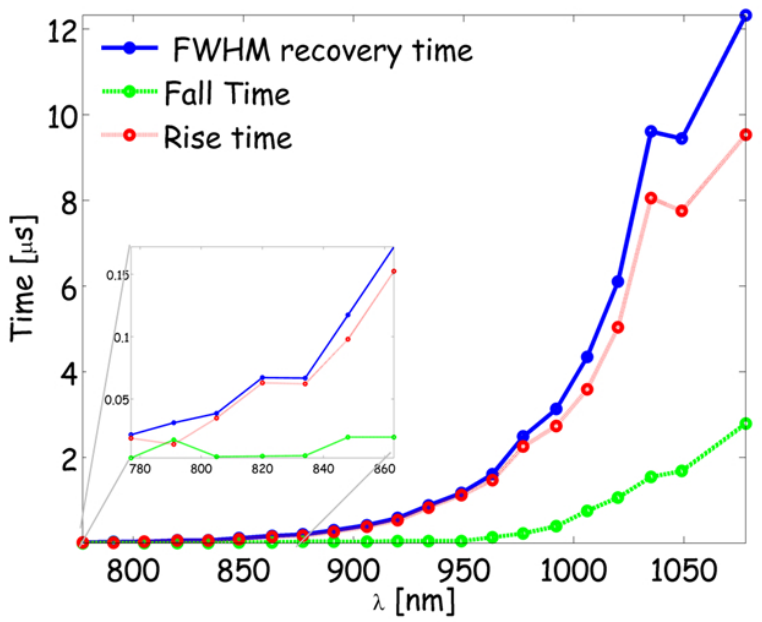

FIG. 4 Experimental results of the FWHM (blue), the fall (green) and the rise time (red) versus the wavelength of the pump.

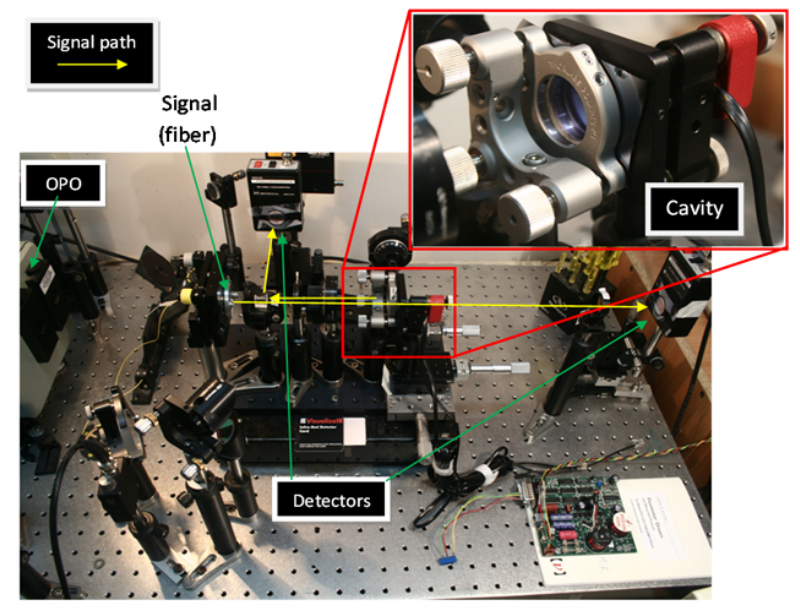

FIG. 5 Photograph of the experimental system. The cavity is placed on an isolated board, and comprised from a silicon polished wafer positioned between two mirrors.

itatively, both the pump penetration depth and the recovery time have similar behavior, and it can be seen that the smaller the penetration depth of the pump is, the shorter is the recovery time of the signal.

This claim is reinforced in Figure 4, where one can see the quick fall time and the correlation between the total recovery of the signal and the rise time.

\subsection{Cavity}

We used a $500 \mu \mathrm{m}$ thick parallel double side polished intrinsic silicon (resistivity $\sim 1700 \Omega \mathrm{cm}$ ) and illuminated it perpendicularly using a narrow band $(100 \mathrm{KHz})$ IR tunable laser (HP 8168 F) followed by EDFA, to realize a F-P cavity similar to the one described in the theoretical section.

We examined three types of cavities with different values for the finesse: bare silicon where the cavity is formed due to Fresnel reflections, bare silicon followed by a single mirror and two mirrors with silicon sample in between. In all experiments the signal was perpendicularly illuminating the constructed 


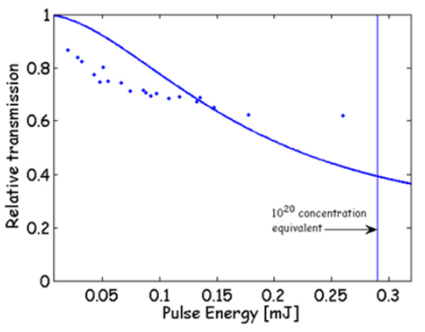

(a).

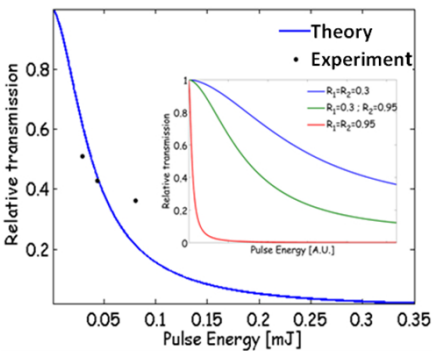

(c).
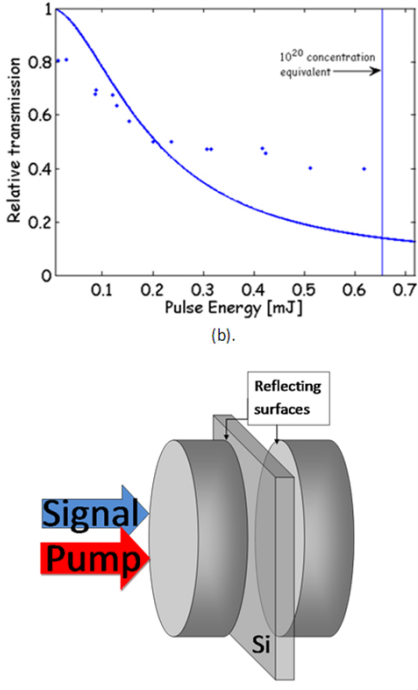

(d).
FIG. 6 Experiential results for relative transmission versus the pulse energy. (a). Theoretical (solid) and experimental (dotted) results of relative transmission of uncoated silicon cavity. The vertical line represents the pulse energy in which the carriers' concentration is $10^{20} \mathrm{~cm}^{-3}$. (b). Silicon sample followed by a $95 \%$ reflecting mirror $(\mathrm{F} \approx 5)$. (c). Silicon sample is inserted into a cavity formed by two mirrors with reflectivity of $95 \%$ each. (d). Schematic sketch of the experimental setup of Figure 6(c).

device in order to get the highest possible finesse, and the mirrors were designed to have high transmission at the pump wavelength (532 nm). An image of the experimental system with high finesse cavity is presented in Figure 5.

The first result we present is related to the relation between the modulation depth and the power of the pump source $(532 \mathrm{~nm})$. The modulation depth is an exponential function of the pump's power, and one can see good agreement between the theoretical calculations and the experimental results seen in Figure 6(a).

At higher energies the relative transmission is a bit higher than the calculation since in these energies the silicon face is optically damaged from the pump pulses. As an outcome a significant fraction of the pump is being scattered and this causes the signal's transmission to be higher than the anticipated values. In addition, the pump beam jitters significantly and the spatial overlap between the pump and the signal beams is constantly changing between pulses. The beam size of the pump was measured using Ophir-Spiricon laser beam analyzer (LBA sp620u) to be $\sim 2.1 \mathrm{~mm}$ in diameter and the average fit to Gaussian is $\sim 0.5$ which means multi-mode laser.

Once we place a 95\% reflecting mirror after the silicon sample we double the finesse and the device became a bit more sensitive. The results are depicted in Figure 6(b) where the theoretical beam diameter was fitted to be $4 \mathrm{~mm}$.

In Figure 6(c) we repeat the same experiment while this time the silicon wafer is inserted into a cavity formed by two mirrors with reflectivity of $95 \%$ each. Figure $6(\mathrm{~d})$ presents the schematic sketch of the experimental setup of Figure 6(c). In the inset of Figure 6(c) we present the theoretical dependence of the relative transmission on the pulse energy for cavity with

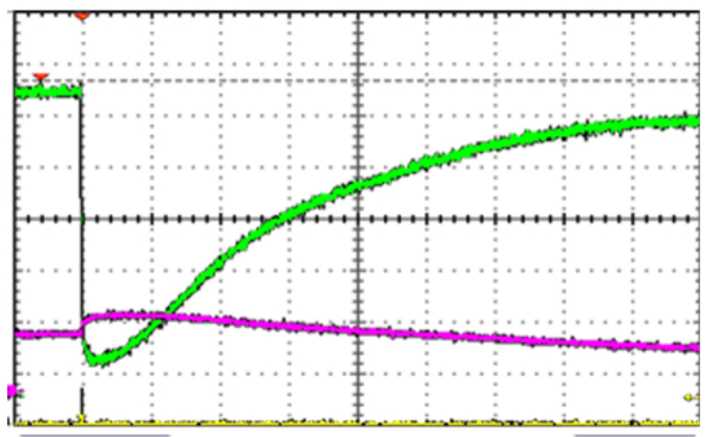

(a).



(b).

FIG. 7 Experimental results. (a). Attenuation of an $1550 \mathrm{~nm}$ signal (green line) in the presence of a $425 \mu$ J pump pulse at wavelength of $532 \mathrm{~nm}$ at time scale of $200 \mathrm{~ns} /$ div. The yellow line is an optical trigger, and the pink is the reflected signal. Introducing the pump pulse shifts the cavity resonance and the transmission drops. (b). Attenuation in non-cavity sample of IR signal (green) in presence of $460 \mu$ J pump pulse at wavelength of $532 \mathrm{~nm}$. The time scale is $40 \mathrm{~ns} /$ div.

different finesse values (different reflectivity coefficients $R_{1}$ and $R_{2}$ for the two mirrors of the cavity). In general in the inset of Figure 6(c) one can see the influence of the pump pulse energy on the modulation depth in three different cavities. As the finesse value rises, less energy is required to get significant modulation. In Figure 6(c) the finesse in the theoretical solid curve is fitted to 30 instead of the theoretical value of 60 (which means $90 \%$ reflection instead of $95 \% \pm 2 \%$ manufacturer specs) to get a good agreement with the experiment, from some reasons to be described.

Figure 6 shows some differences between theoretical and experimental results since in this experiment it is very hard to get the theoretical finesse as this system (comprised of silicon sample positioned between two mirrors) is very unstable due to ambient thermal noise and mechanical vibrations that cause constant movement of the transmission function of the cavity. A controlled ambient temperature and better vibration isolation could improve the cavity stability. The mirrors separation was kept minimized to $\sim 1 \mathrm{~mm}$ and the components were assembled using the lowest possible post system, to reduce the influence of vibrations. The mirrors and silicon non-perfect parallelism and surface quality, as well as issues related to misalignment of the cavity are also optional reasons for the drop down of the finesse in respect to its theoretical values.

As stated before, placing the sample between two parallel mirrors with $95 \%$ reflectivity at $1550 \mathrm{~nm}$ and anti reflection (AR) coating for $532 \mathrm{~nm}$ produced a theoretically high finesse cavity having finesse of about 60. In Figure 7(a) the wavelength 
of the IR signal is tuned to maximal transmission of the cavity, and by introducing $425 \mu \mathrm{J}$ pump pulse this resonant transmission peak is swiped to reduce the overlap with the signal which experienced a drop in its transmission to $\sim 9 \%$ of the signal's value before applying the pump pulse.

The recovery time of the signal is longer than in the noncavity case (Figure 7(b)) due to thermal effects that are also reinforced when cavity is involved (thermal effects may cause changes in the average refraction index of the sample and change the signal's phase). The reflection is not close to zero since the IR signal is amplified with EDFA which produces a considerable amount of amplified spontaneous emission (ASE) that is being reflected. This reflected ASE is a broadband signal and most of its spectrum cannot obey the resonant condition and therefore most of its energy is being reflected. For that very reason the transmission cannot drop to zero.

For comparison, in Figure 7(b) we used a sample of the same kind and polished it to be wedged in order to prevent cavity formation. In this case a $460 \mu \mathrm{J}$ pump pulse results with $80 \%$ transmission of the signal, which is 9 times higher than in the previous case of Figure 7(a).

\subsection{Thermo-optical effect}

To investigate and approve the calculations in Section 2.3 we measured the reflection of the CW IR $1550 \mathrm{~nm}$ laser from a $500 \mu \mathrm{m}$ thick bare silicon cavity in different temperatures. The cavity was placed between two copper plates, due to its high heat conductivity, surrounded by ceramic resistors acting as heaters. The current through the resistors was controlled by a temperature controller and thermo-couple. On the cavity itself a calibrated resistor (Minco PT100 temperature probe) was attached to the silicon in a close proximity to the laser spot which was passing through a small hole in the copper plates acting as a thermometer.

By changing the temperature of the sample we control the refractive index and the phase which results in a different reflection as depicted in Figure 8. The solid red curve represents the calculated reflection on a cavity over a phase of $2 \pi$ while the blue line is the measured normalized intensity of a single wavelength in different temperatures. A temperature difference of $>3^{\circ} \mathrm{C}$ is required for a $\pi$ phase shift.

\subsection{Waveguide}

The experiments above are a proof of concept to a possibility of generating a silicon cavity based fiber integrated modulator. One of our future activities is to convert the concept demonstrated in this paper into a compact all-fiber integrated modulator in which the free space cavity is replaced with a section of the fiber is constructed from a silicon core waveguide surrounded by a silica cladding. The cavity itself can be realized by recording Bragg mirrors on both sides of the silicon core, separated axial distance of $10 \mu \mathrm{m}$ (from Figure 2 we see that in order to achieve fast operation rates for long pump wavelength one needs that the thickness of the silicon layer will be smaller than $20 \mu \mathrm{m})$.

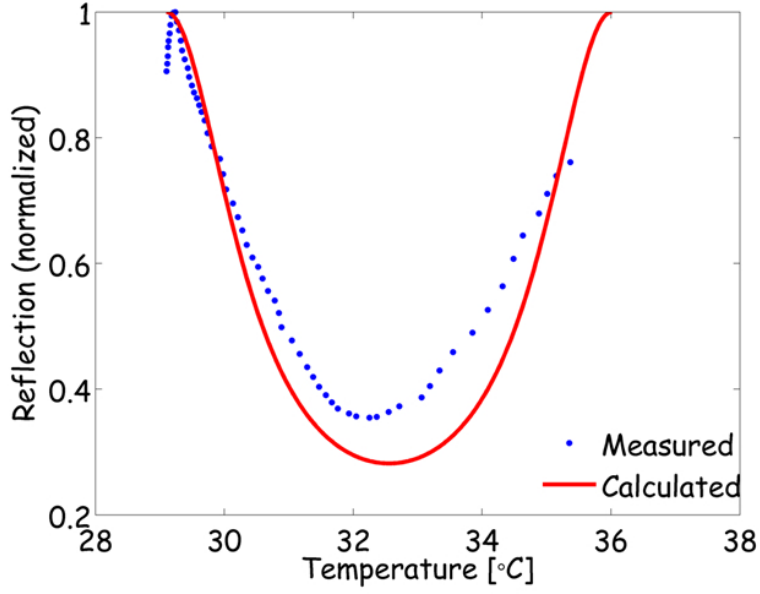

FIG. 8 Silicon cavity under the influence of TOE. Red solid line represents calculated normalized intensity over $2 \pi$. Blue dots represent measured normalized intensity.

The pump and the signal both will be inserted to a single fiber through a directional $Y$ coupler and then edge of the fiber will be coupled to the above mentioned silicon waveguide with cross-section being large enough to guide the signal without any nonlinear effects.

Since currently our fabrication capabilities cannot provide high quality silicon-core fibers, we designed and fabricated a preliminary device as a proof of concept: a $10 \mu \mathrm{mX} 10 \mu \mathrm{m}$ buried silicon waveguide surrounded with silicon having lower refraction index and acting as a clad. An n-type silicon wafer with resistivity of $0.02 \Omega \mathrm{cm}$ is used as a clad, upon which grooves were etched (using deep RIE process) and an epilayer of intrinsic silicon $\left(\sim 10^{14} \mathrm{~cm}^{-3}\right)$ was deposited on top, with thickness of $20 \mu \mathrm{m}$ to fill the grooves. We polished an excess of $10 \mu \mathrm{m}$ of the epilayer to form a rectangular waveguide with refractive index of $n=3.5$ surrounded by a clad with $n=3.498$. Due to the impurities of the clad this is a lossy waveguide but as shown above, when using a proper pump wavelength only a short section is required in order to produce significant attenuation.

In Figure 9(a) we simulate the propagated electrical field at wavelength of $1550 \mathrm{~nm}$ using Rsoft software. The launched field is the first mode (mode 0) waveguide. This one was calculated using a semi-vectorial method for which the polarization is either TE or TM. The simulations we performed gave the same results for the two polarizations. The light is propagated in such a waveguide and one can see insignificant attenuation after $1 \mathrm{~cm}$ of propagation, as the attenuation is only $1.2 \mathrm{~dB} / \mathrm{m}$. In Figure 9(b) we present an image of the fabricated waveguide device (silicon with different values of doping for core and for cladding) as it was obtained using scanning electron microscope (SEM). The fabricated silicon core is in the center of the image and it is bounded with white lines.

\section{CONCLUSIONS}

In this paper we have presented an all-optical silicon based modulator suggested for the pulse picker application to be used as part of fiber lasers system operating at power levels 


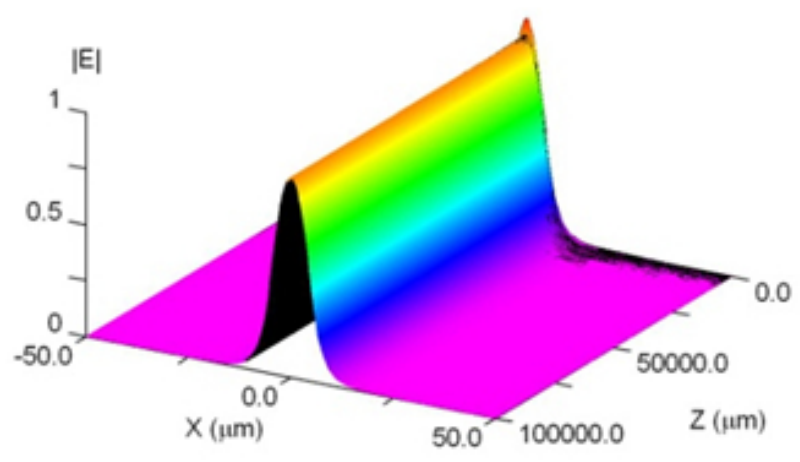

(a).

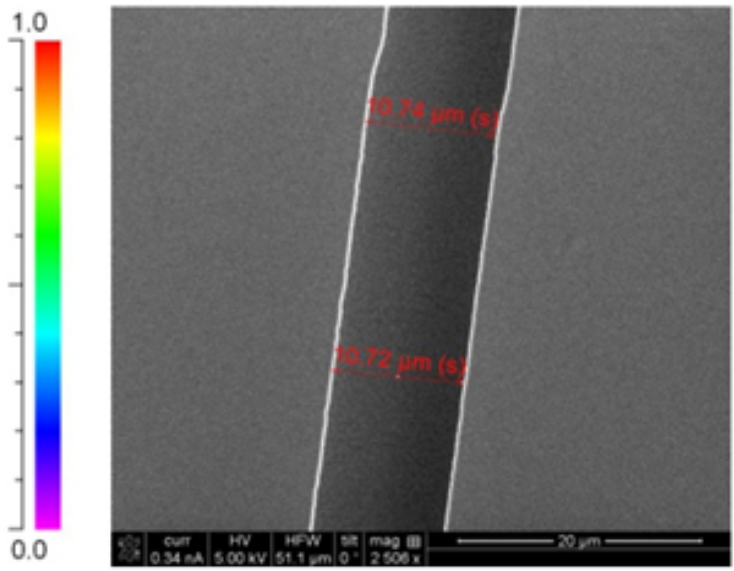

(b).

FIG. 9 Waveguide. (a). Numerical simulation of the cross-section of the electrical field at wavelength of $1550 \mathrm{~nm}$. The signal is guided in a silicon waveguide having square cross section. The amplitude of the electrical field was measured in the XZ plane (Z being the propagation axis). (b). SEM image of the fabricated silicon waveguide.

above the level typically used in optics communication. The paper includes several insights that were experimentally investigated and theoretically supported: We theoretically and experimentally investigated the dependence of the response rate on the pump wavelength (we were able to separate between the fast surface effect and the slower bulk process) and the dependence of the relative transmission on the pump pulse energy for different finesse values of the constructed Fabry-Perot cavity. Although we used relatively low power some considerations were made for the high power application. It has also been showed that the TOE becomes more significant as the finesse of the cavity rises.

To make the proposed cavity based all-optical configuration suitable for high power fiber laser application we succeeded in aligning the pump and the signal beams to co-propagate through the device while the cavity allowed low power pump to generate significant extinction ratio.

Foundations for future work of integrating this device as a waveguide coupled to fibers is presented as well.

\section{References}

[1] R. A. Soref, and B. Bennett, "Electrooptical effects in silicon," IEEE J. Quantum Elect. 23, 123-129 (1987).

[2] S. Stepanov, and S. Ruschin, "Modulation of light by light in siliconon-insulator waveguides," Appl. Phys. Lett. 83, 5151-5153 (2003).

[3] A. Liu, R. Jones, L. Liao, D. Samara-Rubio, D. Rubin, 0. Cohen, R. Nicolaescu, and M. Paniccia, "A high speed silicon optical modulator based on a metal-oxide-semiconductor capacitor," Nature 427, 615-618 (2004).

[4] L. Liao, D. Samara-Rubio, M. Morse, A. Liu, D. Hodge, D. Rubin, U. D. Keill, and T. Franck, "High speed silicon Mach-Zehnder modulator," Opt. Express 13, 3129-3135 (2005).

[5] V. M. N. Passaro, and F. Dell'Olio, "Scaling and Optimization of MOS Optical Modulators in Nanometer SOI Waveguides," IEEE T. Nanotechnol. 7, 401-408 (2008).
[6] C. A. Barrios, V. R. Almeida, and M. Lipson, "Low-powerconsumption short-length and high-modulation-depth silicon electrooptic modulator," J. Lightwave Technol. 21, 1089-1098 (2003).

[7] V. R. Almeida, C. A. Barrios, R. R. Panepucci, and M. Lipson, "Alloptical control of light on a silicon chip," Nature 431, 1081-1084 (2004).

[8] S. Schönenberger, T. Stöferle, N. Moll, R. F. Mahrt, M. S. Dahlem, T. Wahlbrink, J. Bolten, et.al, "Ultrafast all-optical modulator with femtojoule absorbed switching energy in silicon-on-insulator," 0 pt. Express 18, 22485-22496 (2010).

[9] G. T. Reed, G. Mashanovich, F. Y. Gardes, and D. J. Thomson, "Silicon optical modulators," Nat. Photonics 4, 518-525 (2010).

[10] S. Preble, Q. Xu, B. Schmidt, and M. Lipson, "Ultrafast all-optical modulation on a silicon chip," Opt. Lett. 30, 2891-2893 (2005).

[11] K. Narayanan, A. Elshaari, and S. Preble, "Broadband all-optical modulation in hydrogenated-amorphous silicon waveguides," 0pt. Express 18, 9809-9814 (2010).

[12] A. Kumar, and V. Rastogi, "Design and analysis of dual-shape-core large-mode-area optical fiber," Appl. Optics 50, 119-124 (2011).

[13] C. Wirth, 0. Schmidt, A. Kliner, T. Schreiber, R. Eberhardt, and A. Tünnermann, "High-power tandem pumped fiber amplifier with an output power of 2.9kW," Opt. Lett. 36, 3061-3063 (2011).

[14] K. Danekar, A. Khademian, and D. Shiner, "Blue laser via IR resonant doubling with $71 \%$ fiber to fiber efficiency," Opt. Lett. 36, 2940-2942 (2011).

[15] R. Zhou, Y. Ju, Y. Zhang, and Y. Wang, "High-powered millijoule pulse energy Tm3+-doped fiber amplifier at $2.05 \mu \mathrm{m}$," Chin. 0pt. Lett. 9, 071401-071401 (2011).

[16] P. Hübner, C. Kieleck, S. D. Jackson, and M. Eichhorn, “Highpower actively mode-locked sub-nanosecond Tm3+-doped silica fiber laser," Opt. Lett. 36, 2483-2485 (2011).

[17] D. B. S. Soh, S. E. Bisson, B. D. Patterson, and S. W. Moore, "Highpower all-fiber passively Q-switched laser using a doped fiber as a saturable absorber: numerical simulations," 0pt. Lett. 36, 2536-2538 (2011).

[18] M. Gorjan, R. Petkovšek, M. Marinček, and M. Čopič, "High-power pulsed diode-pumped Er:ZBLAN fiber laser," Opt. Lett. 36, 1923-1925 (2011). 
[19] E. Gaubas, and J. Vanhellemont, "A simple technique for the separation of bulk and surface recombination parameters in silicon," J. Appl. Phys. 80, 6293 (1996).

[20] G. Coppola, A. Irace, G. Breglio, and A. Cutolo, "All-silicon modemixing router based on the plasma-dispersion effect," J. Opt. A-Pure Appl. 0p. 3, 346-354 (2001).

[21] Q. Xu, B. Schmidt, S. Pradhan, and M. Lipson, "Micrometer-scale silicon electro-optic modulator," Nature 435, 325-327 (2005).

[22] T. S. Moss, G. J. Burrell, and B. Ellis, Semiconductor OptoElectronics (Butterworths, London, 1975).

[23] R. A. Soref, and B. R. Bennett, "Kramers-Kronig analysis of electrooptical switching in silicon," Proc. SPIE 704, 32-37 (1987).

[24] B. Jalali, and S. Fathpour, "Silicon Photonics," J. Lightwave Technol. 24, 4600-4615 (2006).

[25] M. Dinu, F. Quochi, and H. Garcia, "Third-order nonlinearities in silicon at telecom wavelengths," Appl. Phys. Lett. 82, 2954-2956 (2003).

[26] R. A. Soref, and P. J. Lorenzo, "All-silicon active and passive guided-wave components for $\lambda=1.3$ and $1.6 \mu \mathrm{m}$," IEEE J. Quantum Elect. 22, 873-879 (1986).

[27] S. M. Ryvkin, Photoelectric Effects in Semiconductors (Consultants Bureau, New York, 1964).

[28] R. A. Smith, Semiconductors (Cambridge University Press, Cambridge, 1978).

[29] V. R. Almeida, C. A. Barrios, R. R. Panepucci, and M. Lipson, "Alloptical control of light on a silicon chip," Nature 431, 1081-1084 (2004).
[30] K. Preston, P. Dong, B. Schmidt, and M. Lipson, "High-speed all-optical modulation using polycrystalline silicon microring resonators," Appl. Phys. Lett. 92, 15-17 (2008).

[31] A. Chin, K. Y. Lee, B. C. Lin, and S. Horng, "Picosecond photoresponse of carriers in Si ion-implanted Si," Appl. Phys. Lett. 69, 653-655 (1996).

[32] B. Cowan, "Optical Damage Threshold of Silicon for Ultrafast Infrared Pulses," AIP Conf. Proc. 877, 837-843 (2006).

[33] J. R. Chelikowsky, and M. I. Cohen "Electronic structure of silicon," Phys. Rev. B 10, 5095-5107 (1974).

[34] H. K. Tsang, C. S. Wong, T. K. Liang, I. E. Day, S. W. Roberts, A. Harpin, J. Drake, and M. Asghari, "Optical dispersion, two-photon absorption and self-phase modulation in silicon waveguides at $1.5 \mu \mathrm{m}$ wavelength," Appl. Phys. Lett. 80, 416-418 (2002).

[35] X. Wang, Z.H. Shen, J. Lu, and X.W. Ni, "Laser-induced damage threshold of silicon in millisecond, nanosecond, and picosecond regimes," J. Appl. Phys. 108, 033103 (2010).

[36] G. Cocorullo, and I. Rendina, "Thermo-optical modulation at $1.5 \mu \mathrm{m}$ in a silicon etalon," Electron. Lett. 28, 83-85 (1992).

[37] S. Manipatruni, R. K. Dokania, B. Schmidt, N. Sherwood-Droz, C. B. Poitras, A. B. Apsel, and M. Lipson, "Wide temperature range operation of micrometer-scale silicon electro-optic modulators," Opt. Lett. 33, 2185-2187 (2008).

[38] M. A. Green, and M. J. Keevers, "Optical properties of intrinsic silicon at 300 K," Prog. Photovoltaics 3, 189-192 (1995). 